\title{
Clinical Efficacy and Prognostic Factors in Locally Advanced Cervical Cancer Patients Treated With Concurrent Chemoradiotherapy
}

\author{
Hui Xiong ${ }^{1}$, Ping Liu ${ }^{1}$, Wen Zou ${ }^{1}$, Chunhong $\mathrm{Hu}^{1 *}$ \\ ${ }^{1}$ Department of Oncology, the Second Xiangya Hospital of Central South \\ University, Changsha, Hunan 410011, P.R. China.
}

*Correspondence: Chunhong Hu, Email: huchunh@medmail.com.cn.

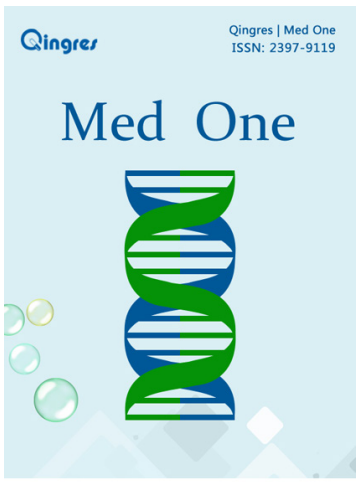

http://mo.qingres.com

\section{GOPEN ACCESS}

DOI: $10.20900 / \mathrm{mo} .20170004$

Received: December 2, 2016

Accepted: January 22, 2017

Published: February 25, 2017

Copyright: ๑2017 Cain et al. This is an open access article distributed under the terms of the Creative Commons Attribution License, which permits unrestricted use, distribution, and reproduction in any medium, provided the original author and source are credited.

\begin{abstract}
Objective: To analyze the clinical efficacy and toxic \& adverse effects of radiotherapy combined with concurrent chemoradiotheray on locally advanced cervical cancer patients, and analyze the prognostic factors.

Methods: The clinical data of 125 patients with locally advanced cervical cancer who underwent radiotherapy and concurrent chemoradiotherapy between September 2008 and December 2013 were retrospectively analyzed in the Second Xiangya Hospital of Central South University. Kaplan-Meier method was used to calculate the survival rate. In univariate analysis, log-rank test was used to calculate the intergroup difference, and in multivariate analysis, Cox proportional risk model was used to screen the independent prognostic factors influencing the overall survival rate.
\end{abstract}

Results: As of March 1, 2016, the overall survival rates of the patients were $89.6 \%, 74.4 \%$ and $71.2 \%$ in years 1,3 and 5 , respectively. The results of univariate analysis showed that the survival rates of stageII, stage-III and stage-IVa patients for PIGO staging were $92.6 \%$, $79.4 \%$, and $77.9 \%$; $88.7 \%, 66.0 \%$, and $64.2 \%$; and $50.0 \%, 25.0$ $\%$, and $0.0 \%$ in years 1,3 and 5 , respectively. The overall survival rates of the patients in concurrent chemoradiotherapy group and radiotherapy group were $91.8 \%, 78.6 \%$, and $77.6 \%$; $81.5 \%, 61.2$ $\%$, and $58.5 \%$ in years 1,3 and 5 , respectively. The overall survival rates of the patients with pre-treatment hemoglobin $\mathrm{Hb}$ level $<110 \mathrm{~g} /$ $\mathrm{L}$ and $\mathrm{Hb} \geq 110 \mathrm{~g} / \mathrm{L}$ were $78.4 \%, 59.8 \%$, and $58.2 \%$; $92.9 \%, 79.8$ $\%$, and $78.9 \%$ in years 1,3 and 5 , respectively. The overall survival rates of the patients with total radiotherapy duration $\leq 9$ cycles and $>$ 9 cycles were $91.2 \%, 81.7 \%$, and $80.0 \% ; 87.8 \%, 64.6 \%$, and 59.8 $\%$ in years 1,3 and 5 , respectively. These differences were statistically significant. Multivariate analyses showed that the clinical staging, concurrent chemoradiotherapy, pretreatment hemoglobin $\mathrm{Hb}$ level, and radiotherapy duration were the independent prognostic factors significantly affecting the survival rate of patients with locally advanced 
cervical cancer.

Conclusion: 1 . the concurrent chemoradiotherapy of locally advanced cervical cancer patients showed more obvious advantages over the simple radiotherapy, and the acute toxicity was tolerable and the long-term toxicity was not significantly increased; 2. FIGO staging, anemia and radiotherapy completion time were the important prognostic factors affecting the treatment of patients with locally advanced cervical cancer.

Key Words: cervical cancer; radiotherapy; concurrent chemoradiotherapy; efficacy and prognosis

\section{INTRODUCTION}

Cervical cancer is a common malignant tumor in female genital tract, and its incidence or morbidity accounts for more than half of malignant tumors in female genital tract, which severely threatens the health and life of the women. In China, about 3-5 million women died of cervical cancer each year ${ }^{[1]}$. The incomplete popularity of anti-cancer screening caused a higher incidence of cervical cancer in rural area than in cities, and this part of patients had often developed into the locally advanced cervical cancer (stage-Ilb - stage-IVa) in their initial treatment ${ }^{[2]}$. This shows that cervical cancer is still a major public health problem. Since the National Cancer Institute of the United States (NCI) developed a cisplatin-based concurrent chemoradiotherapy as the treatment solution of the locally advanced cervical cancer in 1999, the treatment efficacy of cervical cancer has been gradually improved, and the concurrent chemoradiotherapy has nowadays become the main treatment method for advanced cervical cancer in China. Despite a huge success, more than half of stage-III and stage-IV patients of cervical cancer in the clinical practice cannot survive for five years in spite of the best treatment. In this paper, the clinical data of 125 patients with locally advanced cervical cancer underwent radiotherapy and concurrent chemoradiotherapy treated in our department were retrospectively analyzed, the clinical efficacy and survival \& prognosis of the patients treated with radiotherapy and concurrent chemoradiotherapy were summarized, and meanwhile, the prognostic factors under the mode of concurrent chemoradiotherapy were discussed.

\subsection{Basic Information of Patients}

Inclusion criteria: 1) patients for initial treatment; 2) patients with cervical squamous cell carcinoma, adenocarcinoma or adenosquamous carcinoma through pathological diagnosis of cervical living tissue biopsy; 3) patients determined by two clinical gynecologists as FIGO staging, including stageIla, but not suitable for surgery; 4) complete clinical and pathological data. Exclusion criteria: 1) patients concurrently with a history of other tumor diseases; 2) patients associated with other rare pathological types of cervical cancers, like clear cell carcinoma; 3) patients with a history of pelvic radiotherapy or systemic chemotherapy.

The enrolled cases were 125 patients with locally advanced cervical cancer in the tumor center of the Second Xiangya Hospital of Central South University between September 2008 and December 2013 who admitted to pathological diagnosis and chemoradiotherapy. Their clinical and pathological data are detailed in Table 1.

Table 1. Clinical and Pathological Data $(n=125)$

\begin{tabular}{lccc}
\hline Variable & Assignment & Number of Cases & Percentage \\
\hline \multirow{2}{*}{ Age } & $<40$ years old & 9 & $7.2 \%$ \\
& $40-60$ years old & 33 & $66.4 \%$ \\
& $>60$ years old & 33 & $26.4 \%$ \\
FIGO clinical staging & II & 68 & $54.4 \%$ \\
& III & 53 & $42.4 \%$ \\
\hline
\end{tabular}




\begin{tabular}{|c|c|c|c|}
\hline \multirow{3}{*}{ Pathological type } & Squamous cell carcinoma & 115 & $92.0 \%$ \\
\hline & Adenocarcinoma & 8 & $6.4 \%$ \\
\hline & Adenosquamous carcinoma & 2 & $1.6 \%$ \\
\hline \multirow{3}{*}{ Tumor Size } & $<4 \mathrm{~cm}$ & 62 & $49.6 \%$ \\
\hline & $4-6 \mathrm{~cm}$ & 59 & $47.2 \%$ \\
\hline & $>6 \mathrm{~cm}$ & 4 & $3.2 \%$ \\
\hline \multirow{3}{*}{ Differentiation } & Low & 34 & $27.2 \%$ \\
\hline & Medium & 83 & $66.4 \%$ \\
\hline & High & 8 & $6.4 \%$ \\
\hline Pre-treatment hemoglobin & $\mathrm{Hb}<110 \mathrm{~g} / \mathrm{L}$ & 54 & $43.2 \%$ \\
\hline level & $\mathrm{Hb} \geq 110 \mathrm{~g} / \mathrm{L}$ & 71 & $56.8 \%$ \\
\hline \multirow{2}{*}{ Lymph node metastasis } & NO & 117 & $93.6 \%$ \\
\hline & YES & 8 & $6.4 \%$ \\
\hline \multirow{2}{*}{ Total radiotherapy duration } & $\leq 9$ weeks & 35 & $28.0 \%$ \\
\hline & $>9$ weeks & 90 & $72.0 \%$ \\
\hline \multirow{2}{*}{$\begin{array}{l}\text { Intracavitary radiotherapy } \\
\text { source intensity }\end{array}$} & $\leq 5 \mathrm{ci}$ & 73 & $58.4 \%$ \\
\hline & $>5 \mathrm{ci}$ & 52 & $41.6 \%$ \\
\hline \multirow{2}{*}{$\begin{array}{l}\text { Concurrent chemotherapy or } \\
\text { not }\end{array}$} & RT & 27 & $21.6 \%$ \\
\hline & CCRT & 98 & $78.4 \%$ \\
\hline
\end{tabular}

\subsection{Radiotherapy Solution}

$6 \mathrm{MV}-\mathrm{X}$ ray hexagonal large field radiotherapy (30GY/2gy/15F) and then pelvis front-back fourfield wear irradiation (middle $4 \times 15 \mathrm{~cm}$ lead block) to $50 \mathrm{GY} / 2 \mathrm{GY} / 25 \mathrm{~F}$; IIIB was appropriately added to $56 \mathrm{GY} / 2 \mathrm{GY} / 28 \mathrm{~F}$; for the patients with abdominal aortic lymph node metastasis, abdomen para-aortic field radiotherapy was increased, with the dosage of 40-50Gy.

Iridium 192 source y rays with intracavitary brachtherapy dosage reference point: point $A$. Dosage: $600 \mathrm{c}$ Gy/time. Cumulative dosage of conventional therapy plus intracavitary brachtherapy was about 4200 c Gy/7 times. Intracavitary brachtherapy was operated after the end of the first-stage in vitro radiotherapy, with the frequrency of one time per week. The in vitro radiotherapy and intracavitary brachtherapy were performed alternately.

\subsection{Concurrent Chemotherapy}

The solution of platinum-based two drugs was used for the synchronous chemotherapy: Docetaxel 75 
$\mathrm{mg} / \mathrm{m} 2 \mathrm{~d} 1$ or paclitaxel $135 \mathrm{mg} / \mathrm{m} 2 \mathrm{~d} 1$ for intravenous infusion; nedaplatin $40 \mathrm{mg} / \mathrm{m} 2 \mathrm{~d} 1-3$, carboplatin $\mathrm{d} 1$, and AUC value of 5 for intravenous infusion; the interval of conventional chemotherapy was 21 days per cycle, and lasted for 4-6 cycles.

\subsection{Follow-up Visit}

All patients were advised to persist in the outpatient reviews or the subsequent visits, and receive the regular follow-up visits after they discharged. The overall survival time of a patient refers to the number of interval months from the date of admission in the hospital to the last follow-up date or the death date of the patient.

\subsection{Statistical Methods}

In this study, SPSS17.0 statistical software package was used for the data statistics. Univariate and multivariate analysis methods were used to analyze the prognosis of cervical cancer and factors. Survival rate was calculated using Kaplan-Meier method.
In the univariate analysis, log-rank test was used to calculate the intergroup differences, and in the multivariate analysis, Cox proportional risk model was used to screen the independent prognostic factors affecting the overall survival rate.

\section{RESULTS}

\subsection{Survival Rate of the Whole Group}

The follow-up visits were ended by March 1, 2016, and 125 patients in all were included and persisted in the follow-up visits. By the date of the last followup visit, the follow-up visits were not completed for four patients, and the median follow-up time was 39.6 months. The follow-up visit was ended by March 1, 2016. 89 of 125 patients with cervical cancer survived $(71.2 \%)$, and 36 died (28.8\%). The overall survival (OS) of the whole group was $89.6 \%$, $74.4 \%$, and $71.2 \%$ in years 1,3 and 5 , respectively (see Fig. 1 Overall Survival (\%) of the Whole Group in Five Years )

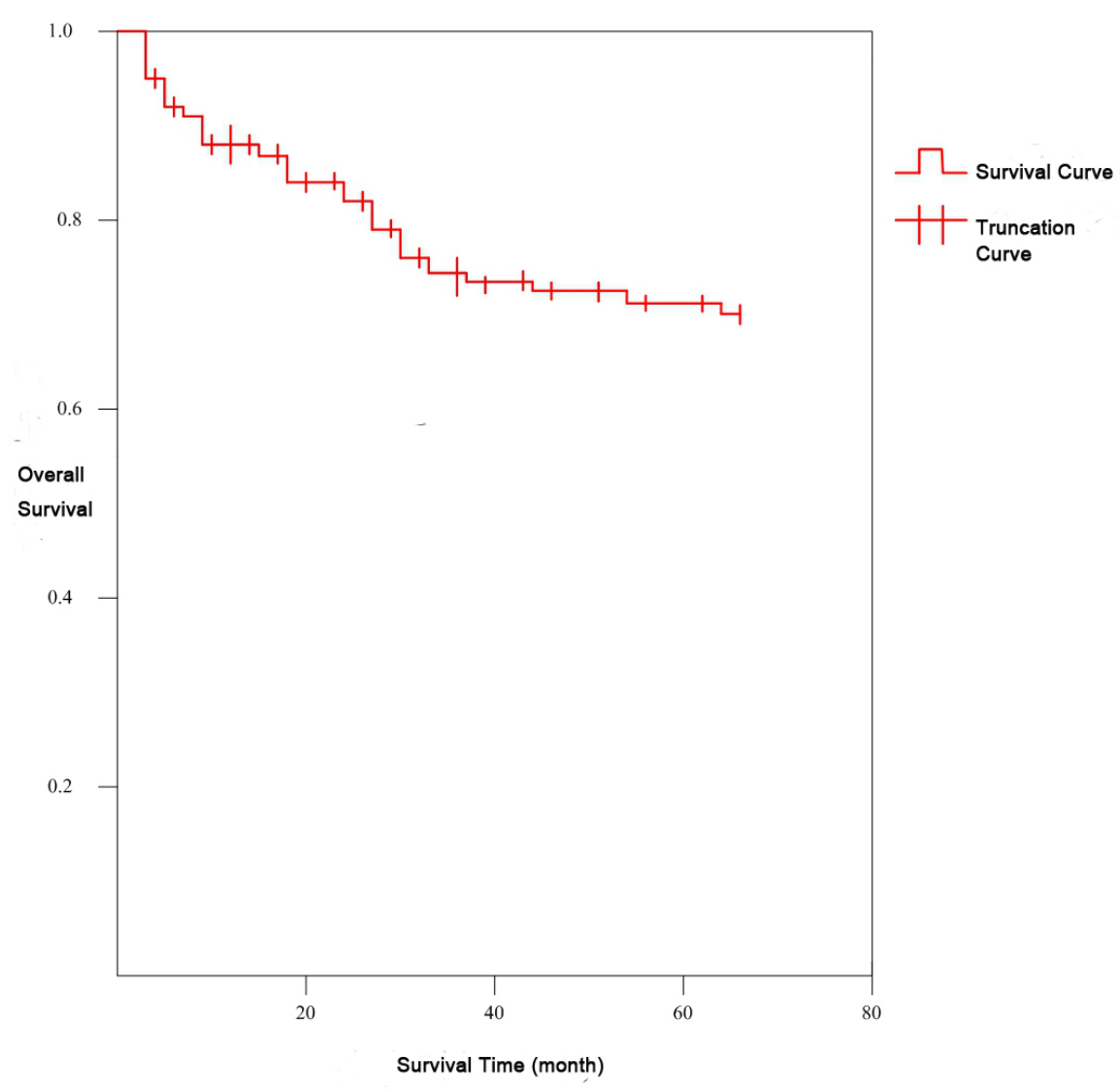

Fig. 1 Overall Survival (\%) of the Whole Group in Five Years 


\subsection{Occurrence of Toxic and Adverse Effect (or Toxicity)}

\subsubsection{Acute toxicity was evaluated as per the}

\section{classification criteria of RTOG acute radiation} injury.

The occurrence condition of the acute toxicity of the patients in this group is shown in Table 2.

Table 2. Acute Toxicity and Classification

\begin{tabular}{|c|c|c|c|c|}
\hline Classification & $\mathbf{N}$ & Incidence & Level & $\mathbf{n}$ \\
\hline \multirow{3}{*}{ Gastrointestinal reaction } & \multirow{3}{*}{49} & \multirow{3}{*}{$39.2 \%$} & Level 1 & 25 \\
\hline & & & Level 2 & 21 \\
\hline & & & Level 3 & 3 \\
\hline \multirow{3}{*}{ Myelosuppression } & \multirow{3}{*}{54} & \multirow{3}{*}{$43.2 \%$} & Level 1 & 31 \\
\hline & & & Level 2 & 17 \\
\hline & & & Level 3 & 6 \\
\hline \multirow{2}{*}{ Rectal reaction } & \multirow{2}{*}{39} & \multirow{2}{*}{$31.2 \%$} & Level 1 & 19 \\
\hline & & & Level 2 & 20 \\
\hline \multirow{2}{*}{ Genitourinary tract reaction } & \multirow{2}{*}{11} & \multirow{2}{*}{$8.8 \%$} & Level 1 & 7 \\
\hline & & & Level 2 & 4 \\
\hline \multirow{2}{*}{ Pelvic effusion } & \multirow{2}{*}{3} & \multirow{2}{*}{$1.6 \%$} & Level 1 & 1 \\
\hline & & & Level 2 & 2 \\
\hline \multirow[b]{2}{*}{ Vaginal inflammation } & \multirow[b]{2}{*}{8} & \multirow[b]{2}{*}{$6.4 \%$} & Level 1 & 7 \\
\hline & & & Level 2 & 1 \\
\hline
\end{tabular}

The incidences of gastrointestinal reaction, myelosuppression, rectal reaction and genitourinary tract reaction were 39.2 $\%, 43.2 \%, 31.2 \%$ and $8.8 \%$ in acute toxicity. The incidences of rectal reaction in the two groups were $25.9 \%(7 / 27)$ and $32.7 \%$ (32/98), respectively. The difference between the two groups was no statistically significant $(p>0.05)$.

2.2.2 Late toxicity was evaluated as per the classification program of RTOG/EORTC late radiation injury.
The occurrence condition of the late toxicity of the patients in this group is shown in Table 3. 
Table 3. Late Toxicity and Classification

\begin{tabular}{lcccc}
\hline Classification & $\mathbf{N}$ & Incidence & Level & Number of cases, $\mathbf{n}$ \\
\hline & & & Level 1 & 4 \\
Radiation enteritis & 6 & $4.8 \%$ & Level 2 & 1 \\
& & & Level 3 & 2 \\
Radiation cystitis & 3 & $2.4 \%$ & Level 1 & 1 \\
Lower-limb venous thrombosis & 1 & & Level 2 & 1 \\
\hline
\end{tabular}

There were 6 cases of radiation enteritis $(4.8 \%, 6 / 125)$, 3 cases of radiation cystitis $(2.4 \%, 3 / 125)$ and 1 case of lowerlimb venous thrombosis resulted from pelvic fibrosis $(0.8 \%)$ in late toxicity. There was no statistical significance in the comparison of groups $(p>0.05)$.

\subsection{Analysis of Prognostic Factors}

\subsubsection{Univariate Analysis}

The univariate analysis of related factors affecting the survival rate of patients with locally advanced cervical cancers in years 1,3 and 5 was performed using the log-rank test. The main analysis parameters include FIGO clinical staging, patient age, pathological type, tumor size, tissue differentiation, pre-treatment hemoglobin level, total radiotherapy duration, intracavitary brachytherapy source intensity and different treatment ways (radiotherapy and concurrent chemoradiotherapy). The analysis results showed that the difference of effects of age, pathological type, tumor size, differentiation and brachytherapy source intensity on the curative efficacy and prognosis was not statistically significant (log-rank, $p>0.05$ ).

\subsubsection{Effect of FIGO staging on treatment efficacy and prognosis}

The survival condition of patients in stage-II $(\mathrm{N}=68)$ group, stage-III ( $\mathrm{N}=53)$ group and stage-IVa $(\mathrm{N}=$ 4) group was compared according to the results of FIGO staging. The comparison results showed that the difference was statistically significant (log-rank, $p=0.006$, see Fig. 2 Overall Survival of Cervical Cancer Patients in Different FIGO Staging (\%) )

\subsubsection{Effect of Concurrent Chemoradiotherapy}

\section{on Curative Efficacy and Prognosis}

Comparison of survival between concurrent chemoradiotherapy and radiotherapy: the concurrent chemoradiotherapy group ( $\mathrm{N}=98)$ had a significant survival advantage over the radiotherapy group ( $\mathrm{N}$ $=27$ ), and the difference was statistically significant (Log-rank, $p=0.012$, see Fig. 3 Overall Survival Rate of Cervical Cancer Patients with Concurrent Chemoradiotherapy (\%) )

\subsubsection{Effect of Anemia on Curative Efficacy and Prognosis}

Cervical cancer anemia determination criteria were defined as $\mathrm{Hb}<110 \mathrm{~g} / \mathrm{L}$. There were 54 cases of patients with anemia before the treatment, accounting for $43.2 \%$. Compared the control group $(\mathrm{N}=71)$, the survival condition of the control group was superior over the anemia group $(\mathrm{N}=54)$, and the difference was statistically significant (Log-rank, $p=0.011$, see Fig. 4. Overall Survival of Anemia Group and Control Group (\%) ).

\subsubsection{Effect of Total Radiotherapy Duration on Curative Efficacy and Prognosis}

Compared with the group with a total radiotherapy duration $>9$ cycles $(N=90)$, the group with a total radiotherapy duration $\leq 9$ cycles $(N=35)$ had a significant advantage of survival, and the difference was statistically significant (log-rank, $p=0.013$, see Fig. 5 Overall Survival of Patients for Different Total Radiotherapy Durations (\%) ). 


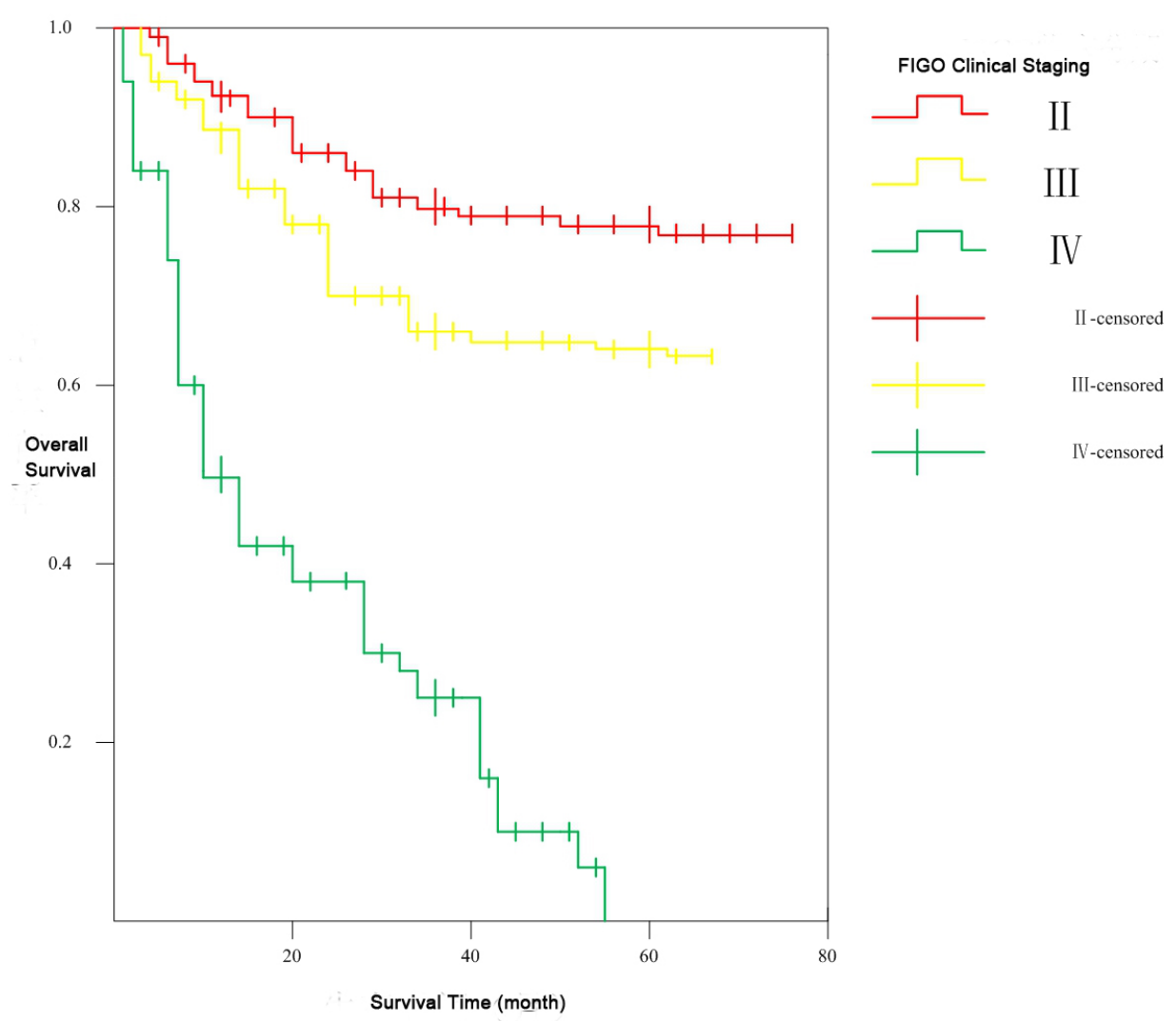

Fig. 2 Overall Survival of Cervical Cancer Patients in Different FIGO Staging (\%)

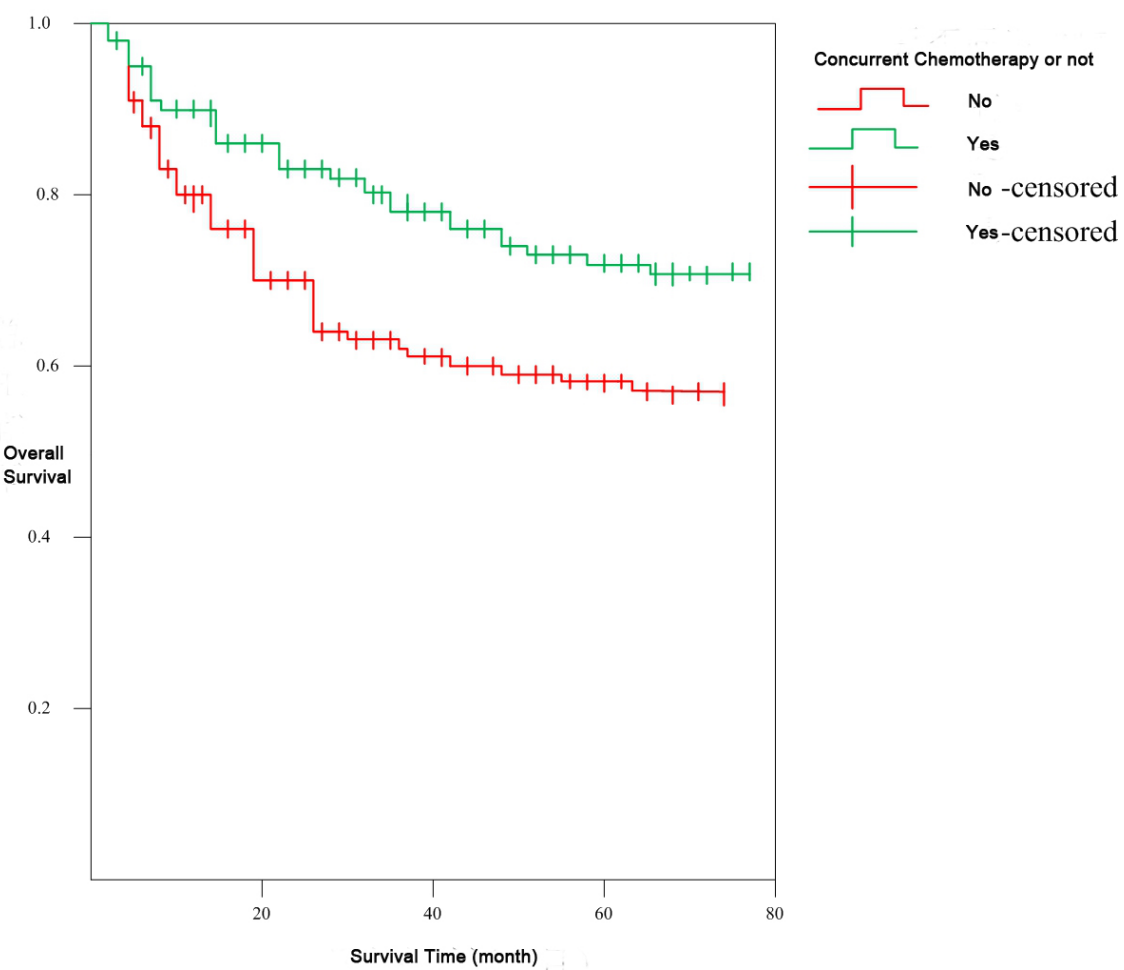

Fig. 3 Overall Survival Rate of Cervical Cancer Patients with Concurrent Chemoradiotherapy (\%) 


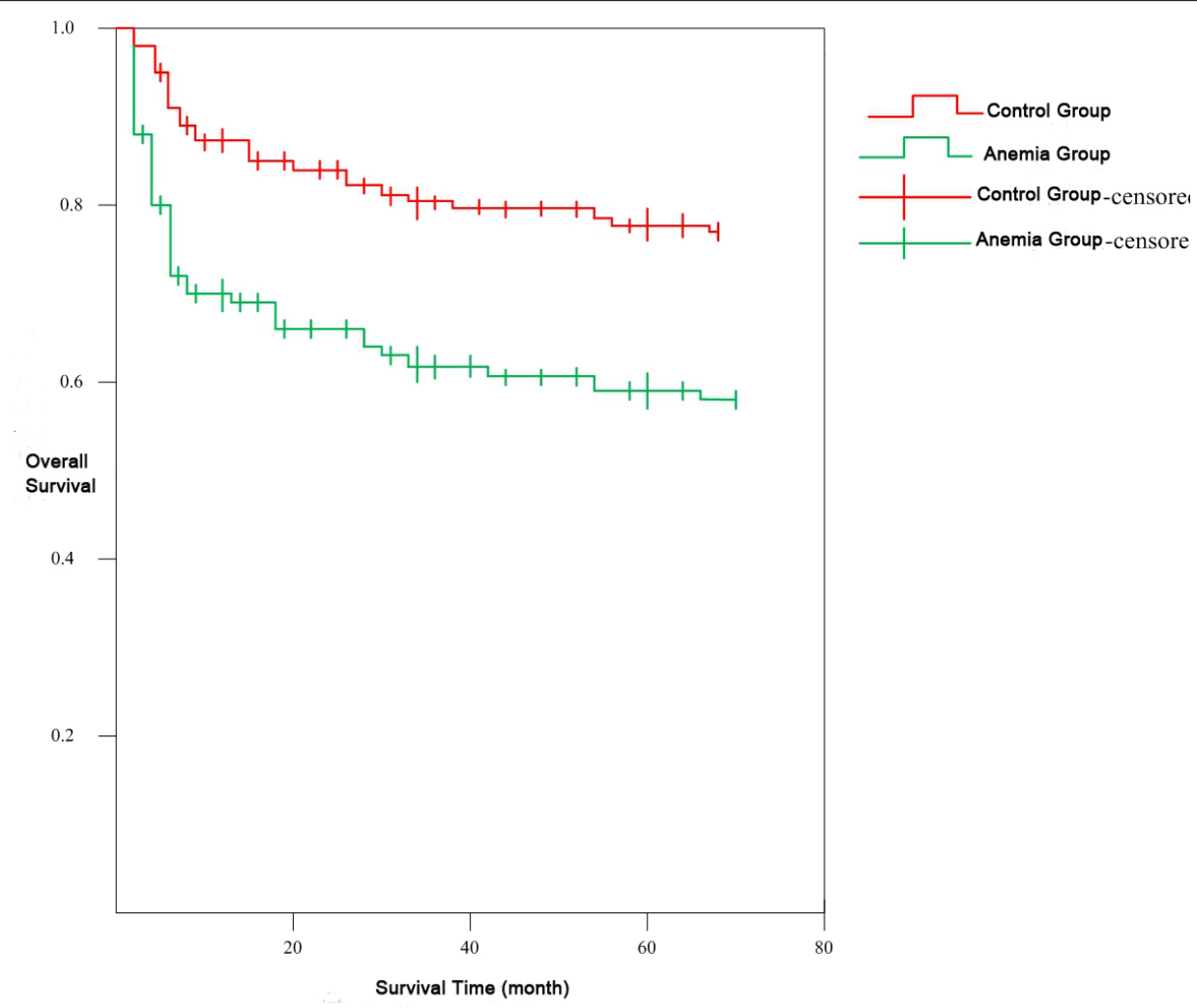

Fig. 4 Overall Survival of Anemia Group and Control Group (\%)

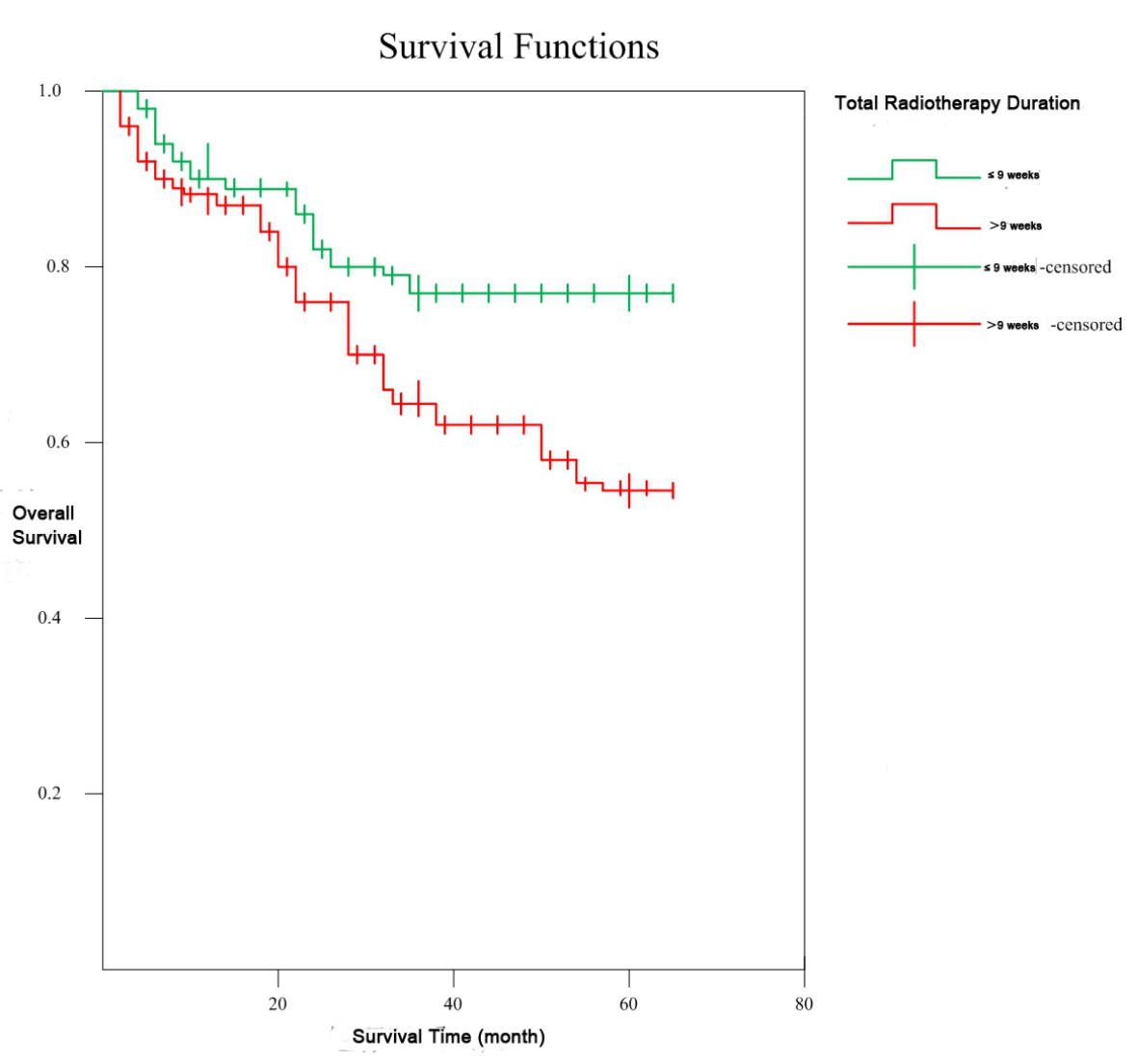

Fig. 5 Overall Survival of Patients for Different Total Radiotherapy Durations (\%) 


\subsubsection{Multivariate Analysis of Prognosis}

Cox proportional risk model was used to screen the independent prognostic factors affecting the overall survival rate. The results showed that FIGO staging, hemoglobin, treatment way and radiotherapy duration were all the independent prognostic factors affecting the locally advanced cervical cancer, especially the hemoglobin and treatment way that had a greater impact on prognosis. (See Table 4).

Table 4. Multivariate Analysis of Survival and Prognosis of 125 Patients with Locally Advanced Cervical Cancer

\begin{tabular}{lcccccc}
\hline Variable & X2 Wald Statistics & P Value & Hazard Ratio & Exp(B) & \multicolumn{2}{c}{$95.0 \%$ Cl for Exp(B) } \\
\hline Clinical staging & 6.064 & 0.010 & 0.329 & 0.106 & 0.770 \\
Hemoglobin & 8.958 & 0.004 & 0.937 & 0.925 & 0.984 \\
Radiotherapy time & 4.080 & 0.025 & 1.081 & 1.022 & 1.095 \\
Treatment way & 9.430 & 0.002 & 0.203 & 0.072 & 0.574 \\
\hline
\end{tabular}

$p<0.05$ was statistically significant.

\section{DISCUSSION}

\subsection{Survival Condition}

$\mathrm{FIGO}^{[3]}$ reported that the survival rates of 10525 cases and 15081 cases of cervical cancer patients treated by it in 1996-1998 and 1999-2001 were 69.9 $\%$ and $69.6 \%$, respectively. Wang Jing, et al. ${ }^{[4]}$ reported that survival rates of 4375 cervical cancer patients in years 1, 3 and 5 were $95 \%, 85 \%$ and $77 \%$, respectively, after the analysis of prognosis and influencing factors, and the median survival time was 175.3 months. In this study, the overall survival rates of locally advanced cervical cancer patients in years 1,3 and 5 were $89.6 \%, 74.4 \%$, and $71.2 \%$, respectively, which are similar to those reported at home and abroad.

\subsection{Related Toxicity (or Toxic and Side Effect)}

Keys, et al. ${ }^{[5]}$ reported that there was an incidence of at least stage-III toxicity in $35 \%$ patients weekly operated for synchronous cisplatin chemoradiotherapy, and most of them were hematological toxicities; GOG-120 ${ }^{[6]}$ also reported the similar proportion of acute complications caused by the weekly operated cisplatin chemoradiotherapy. However, there was few detailed analysis and report about the late complications caused by the concurrent chemoradiotherapy. Eifel and his colleagues ${ }^{[7]}$ reported the similar late toxicities after 6.6 years' median follow-up visit after the concurrent chemoradiotherapy and extended field radiotherapy, which were $13 \%$ and $12 \%$, respectively. Pearcey and his colleagues ${ }^{[8]}$ reported the similar incidence of late toxicities in the patients weekly operated for cisplatin concurrent chemoradiotherapy. In this study, the incidences of gastrointestinal reactions, myelosuppression, rectal reactions, and genitourinary tract reaction in the acute toxicity were 
$39.2 \%, 43.2 \%, 31.2 \%$, and $8.8 \%$, respectively. Among them, the incidences of gastrointestinal reaction and myelosuppression were $14.8 \%(4 / 27)$ and $22.2 \%(6 / 27)$, respectively, in radiotherapy group and they were $45.9 \%$ (45/98) and $48.9 \%$ (48/98), respectively, in concurrent chemoradiotherapy. The difference was statistically significant $(p<0.05)$. The toxicities were mostly occurred in Level 1 and Level 2. The incidences of rectal reactions in the two groups were $25.9 \%(7 / 27)$ and $32.7 \%$ (32/98), respectively, and the most symptoms were increased stool frequency, or acute enteritis accompanied with diarrhea. These symptoms were relieved or mitigated after the symptomatic treatment, and the difference for comparison between the two groups was not statistically significant $(p>0.05)$. The incidences of the genitourinary tract reaction in the two groups were also similar, namely, $7.4 \%(2 / 27)$ and $9.1 \%$ (9/98), respectively, which were all occurred in level 1 and level 2, and the difference not statistically significant $(p>0.05)$.

There were 6 cases of radiation enteritis $(4.8$ $\%, 6 / 125), 3$ cases of radiation cystitis $(2.4 \%$, $3 / 125$ ) and 1 case of lower-limb venous thrombosis resulted from pelvic fibrosis $(0.8 \%)$ in late toxicity, including 1 case of radiation enteritis $(3.7 \%, 1 / 27)$ and 1 case of cystitis $(3.7 \%, 1 / 27)$ in radiotherapy group, and 5 ca ses of radiation enteritis (5.1 $\%, 5 / 98)$ and 3 cases of cystitis $(3.1 \%, 3 / 98)$ in concurrent chemoradiotherapy group, and there was no statistical significance in the comparison of two groups ( $p>0.05$ ). To sum up, the treatment of acute and late toxicities is also one of the main challenges in the treatment of cervical cancer, and its long-term effects shall be further determined via long-term and large-sample studies ${ }^{[9]}$. This is also an important issue.

\subsection{Effect of Related Factors on Survival Prognosis}

Most clinical researchers believe that the FIGO staging has a very important effect on the prognosis of the patients with locally advanced cervical cancer and it is an independent prognostic factor. Federation International of Gynecology and Obstetrics (FIGO) ${ }^{[10]}$ reported that the five-year incidences of patients with cervical cancer were $94 \%$ in stage I, $70 \%$ in stage II, $62 \%$ in stage III, and $17 \%$ in stage IV, respectively. In this study, it was observed that the survival rates of patients with cervical cancer in years 1,3 and 5 were $92.6 \%, 79.4 \%$ and $77.9 \%$ in stage II; $88.7 \%, 66.0 \%$ and $64.2 \%$ in stage III and
$50.0 \%, 25.0 \%$ and $0.0 \%$ in stage IVa. The results showed that the survival rates decreased with the increase of stages; the deviation observed between stage IV and other stages might be associated with the fewer observed cases (only 4 cases). Univariate analysis confirmed that FIGO staging was the important prognostic factor. The multivariate analysis of Cox model also showed that FIGO staging was the independent prognostic factor.

Meta-analysis of concurrent chemoradiotherapy operated in patients with locally advanced cervical cancer showed ${ }^{[11]}$ that the concurrent chemoradiotherapy could improve $6 \%$ of 5 -year survival rate and the death risk also reduced by 19 $\%$ or so. RTOG-9001 clinical trial ${ }^{[12]}$ was performed to compare the survival condition of radiotherapy group and concurrent chemoradiotherapy group combined with cisplatin $+5-\mathrm{FU}$, and the results showed that the 5-year survival rate of patients in radiotherapy group was $58 \%$ and it increased by 15 $\%$ (73 \% VS $58 \%$ ) in concurrent chemoradiotherapy group compared with the radiotherapy group. In this study, the overall survival rates of patients in concurrent chemoradiotherapy group and radiotherapy group in years 1,3 and 5 were 91.8 $\%, 78.6 \%$ and $77.6 \% ; 81.5 \%, 61.2 \%$ and 58.5 $\%$, respectively, which showed that the concurrent chemoradiotherapy could significantly improve the survival condition of patients with locally advanced cervical cancer. Multivariate analysis also showed that the concurrent chemoradiotherapy was an independent prognostic factor of cervical cancer. However, there is still no uniform standard for the way of concurrent chemoradiotherapy. The chemotherapy program, administration mode, drug dosage and the combination of different radiotherapy and chemotherapy modes shall be further studied and confirmed.

Cancer Hospital of Chinese Academy of Medical Sciences ${ }^{[13]}$ researched the effect of pre-treatment $\mathrm{Hb}$ concentration on the survival rate of patients with stage-II and stage-III cervical cancer, and found that the 5-year survival rate of patients with a pretreatment $\mathrm{Hb}$ concentration of over $120 \mathrm{~g} / \mathrm{L}$ was significantly better than those with a pre-treatment $\mathrm{Hb}$ concentration below $80 \mathrm{~g} / \mathrm{L}$, and the overall survival rate exceeded $30 \%$ or so. Grogen ${ }^{[14]}$ retrospectively analyzed the data of 605 cervical cancer patients in seven centers in Canada who underwent the radical radiotherapy, and found that tumor local control rate, disease-free survival rate and overall survival rate were significantly correlated with the minimum $\mathrm{Hb}$ level during the radiotherapy period. In univariate analysis of this study, the survival rates 
of patients with pre-treatment hemoglobin $\mathrm{Hb}<110$ $\mathrm{g} / \mathrm{L}$ and $\mathrm{Hb} \geq 110 \mathrm{~g} / \mathrm{L}$ in years 1,3 and 5 were 78.4 $\%, 59.8 \%$ and $58.2 \%$; $92.9 \%, 79.8 \%$ and 78.9 $\%$, respectively, showing that the survival rate of patients in $\mathrm{Hb}$ control group was significantly better than that in low $\mathrm{Hb}$ group, and this confirmed that the pre-treatment hemoglobin level had an important impact on the prognosis of cervical cancer. In the patients operated for concurrent chemoradiotherapy, the overall survival rates of patients in anemia group in years 1, 3 and 5 decreased by $6.2 \%, 17.4 \%$ and $18.2 \%$, respectively, compared with the control group. In the patients operated for radiotherapy, the overall survival rates of patients in anemia group in years 1,3 and 5 decreased by $8.2 \%, 26.5 \%$ and $20.6 \%$, respectively, compared with the control group. Multivariate analysis of Cox model also showed that the pre-treatment hemoglobin level was the independent prognostic factor of cervical cancer. The anemia patients shall be actively corrected to improve the radioactive resistance of tumor cells, which is an important measure to improve the longterm survival of the patients.

The concurrent chemoradiotherapy is currently the standard treatment method of cervical cancer, so it is very difficult to find the cases of simple radiotherapy and concurrent chemoradiotherapy for one-to-one comparison; the number of cases is not adequate and the observation time is not long enough. The inadequacy of this study is that the cases of intensity-modulated radiotherapy were not included in the analysis.

In summary, the concurrent chemoradiotherapy of locally advanced cervical cancer showed a more significant advantage than the simple radiotherapy, the acute toxicity could be tolerated and longterm toxicity was not significantly increased. FIGO staging, anemia and radiotherapy duration are the important prognostic factors affecting the treatment of patients with locally advanced cervical cancer. We expect that the studies in the future will be more convincing.

\section{REFERENCES}

1. Sun J, Cai S, Gao Y. Gynecological Oncology. Beijing: Peking University Medical Press; 2011; 501, 502, 565-566.

2. Swisher EM, Swensen RE, Greer B, Tamimi H, Goff BA, Garcia R, Koh W. Weekly emcitabine and cisplatin in combination with pelvic radiation in the primary therapy of cervical cancer: a phase I trial of the Puget Sound Oncology Consortium. Gynecol Oncol. 2006; 101(3): 429435.

3. Quinn MA, Benedet JL, Odicino F, Maisonneuve $P$, Beller U, Creasman WT, Heintz AP, Ngan HY, Pecorelli S. Carcinoma of the cervix uteri. Int J Gynaecol Obstet. 2006; 95(Suppl 1): 43-103.

4. Wang J, Xu K, Shi B, Zhang K, Zhou P, Liao $X$, Liu S, Zhang Y. Analysis of Prognosis and Impact Factors of 4374 Patients with Cervical Cancer. China Cancer. 2014; 23(4): 235-238.

5. Kim YS, Shin SS, Nam JH, Kim Y, Kim Y, Kim JH, Choi EK. Prospective randomized comparison of monthly fluorouracil and cisplatin versus weekly cisplatin concurrent with pelvic radiotherapy and high-dose rate brachytherapy for locally advanced cervical cancer. Gynecol Oncol. 2008; 108(1): 195-200.

6. Lorvidhaya V, Chitapanarux I, Sangruchi $S$, Lertsanguansinchai $\mathrm{P}$, Kongthanarat $\mathrm{Y}$, Tangkaratt S, Visetsiri E. Concurrent mitomycin C, 5-fluorouracil, and radiotherapy in the treatment of locally advanced carcinoma of the cervix: a randomized trial. Int J Radiat Oncol Biol Phys. 2003; 55: 1226-1232.

7. Souhami L, Gil RA, Allan SE. A randomized trial of chemotherapy followed by pelvic radiation therapy in stage IIIB carcinoma of the cervix. J Clin Oncol. 1991; 9:970-977.

8. Pearcey R, Brundage M, Drouin P. Phase III trial comparing radical radiotherapy with and without cisplatin chemotherapy in patients with advanced squamous cell cancer of the cervix. J Clin Oncol. 2002; 20: 966-972.

9. King M, McConkey C, Latief TN. Improved survival after concur-rent weekly cisplatin and radiotherapy for cervical carcinoma with assessment of acute and late side-effects. Clin Oncol. 2006; 18(1): 38-45.

10. Benedef JL, Odicino F, Maisonneuve P. Carcinoma of the cervix uteri. Int J Gynaecol Obstet. 2006; 95(Suppl 1): 43-103.

11. Zhang WL, Wang Y. Preoperative radiotherapy and cisplatin concurrent chemotherapy of locally advanced cervical cancer. Guangdong Med J. 2012; 33 (8) : 1146-1148.

12. Morris M, Eifel PJ, Winter K, Levenback C, Grigsby PW, Stevens RE, Rotman M, Gershenson D, Mutch DG. Pelvic radiation 
with concurrent chemotherapy versus pelvic and para-aortic radiation for high-risk cervical cancer: a randomized Radiation Therapy Oncology Group clinical trial. N Engl Med. 1999; 340: 1137-1143.

13. Gu X, Yin W, Liu T, Pan G. Radiation Oncology. 4th ed. Being: Pecking Union Medical College
Press; 2008; 259-261.

14. Grogan M, Thomas GM, Melamed I, Wong FL, Pearcey RG, Joseph PK, Portelance L, Crook J, Jones KD. The important of hemoglobin levels radiotherapy for carcinoma of the cervix. Cancer. 1999; 86 (8) : 1528-1536. 